\title{
From Organisation Oriented Programming to Multi-agent Oriented Programming
}

\author{
Olivier Boissier \\ Ecole Nationale Supérieure des Mines \\ 158 Cours Fauriel, 42100 Saint-Etienne, France \\ Olivier.Boissier@emse.fr
}

\begin{abstract}
Social and organizational aspects of agency have become a major issue in the Multi-Agent Systems (MAS) domain. Recent applications of MAS on Web and Ambient Computing enforce the need of using these dimensions in the programming of MAS. The aim is to ensure the governance of such systems while preserving their decentralization and openness. In this talk, we present how multi-agent organisations provide software abstractions, models and tools that contribute to this aim. We focus on the MOISE framework that we have developped during these last years. This framework proposes an organisation programming language for defining multi-agent organisations, that are managed and supported by organisational artifacts at the system programming level and by organisation-awareness mechanisms at the agent programming level. This framework is included in the JaCaMo platform, integration of Jason Agent Programming Language, CarTaGo environment platform and MOISE. We illustrate different features of Organisation Oriented Programming of MAS using different application examples. We will highlight also how it integrates in a broader perspective of multi-agent oriented programming of decentralized and open systems.
\end{abstract}

